\title{
Pierre Martial Abassolo, Fantastique et littérature africaine contemporaine. Entre rupture et soumission aux schémas occidentaux
}

\section{Carminella Biondi}

\section{(2) OpenEdition \\ Journals}

Edizione digitale

URL: http://journals.openedition.org/studifrancesi/5453

DOI: $10.4000 /$ studifrancesi.5453

ISSN: 2421-5856

\section{Editore}

Rosenberg \& Sellier

\section{Edizione cartacea}

Data di pubblicazione: 1 dicembre 2016

Paginazione: $571-572$

ISSN: 0039-2944

\section{Notizia bibliografica digitale}

Carminella Biondi, «Pierre Martial Abassolo, Fantastique et littérature africaine contemporaine. Entre rupture et soumission aux schémas occidentaux », Studi Francesi [Online], 180 (LX | III) | 2016, online dal 01 janvier 2017, consultato il 18 septembre 2020. URL : http://journals.openedition.org/studifrancesi/ 5453 ; DOI : https://doi.org/10.4000/studifrancesi.5453

Questo documento è stato generato automaticamente il 18 settembre 2020.

\section{c)}

Studi Francesi è distribuita con Licenza Creative Commons Attribuzione - Non commerciale - Non opere derivate 4.0 Internazionale. 


\title{
Pierre Martial Abassolo, Fantastique et littérature africaine contemporaine. Entre rupture et soumission aux schémas occidentaux
}

\author{
Carminella Biondi
}

\section{NOTIZIA}

PIERRE MARTIAL ABASSOLO, Fantastique et littérature africaine contemporaine. Entre rupture et soumission aux schémas occidentaux, préface de Lilyan Kesteloot, Paris, Champion, 2015, $312 \mathrm{pp}$.

1 Lilyan Kesteloot, che ha messo la sua autorità di studiosa al servizio di questo libro, spiega nella prefazione le ragioni d'interesse di un lavoro dedicato alla letteratura fantastica fuori dal contesto occidentale, in paesi africani di lingua francese. Si tratta per ora soltanto di un certo numero di paesi (Cameroun, Mali, Benin, Costa d'Avorio...), ma è un buon viatico per estendere la ricerca all'insieme della narrativa africana. La prefazione che segue, quella in cui l'autore spiega le ragioni della scelta del soggetto e evidenzia le precauzioni da adottare nell'applicare la nozione di fantastico al mondo africano, esordisce in maniera piuttosto sorprendente: «Le fantastique est aujourd'hui un motif littéraire important au regard de la pluralité des études critiques. Il constitue même, comme le dit Jean Baronian "une des lignes les plus riches, les plus fascinantes et les plus nécessaires de la création romanesque"». Il testo, importante, di Baronian (Panorama de la littérature fantastique de langue française) da cui è tratta la citazione è però del 1978, vale a dire di 38 anni fa, e si fatica un po' a considerarlo come uno specchio dell'oggi, anche se diamo per scontato che le sue posizioni siano ancora valide. Questo nulla toglie, tuttavia, alle corrette osservazioni della prefazione e all'interesse del lavoro di Abassolo, che tenta un'analisi molto ampia, se non sul piano geografico almeno su quello teorico, della letteratura fantastica africana di lingua francese, in 
chiave comparatistica con lo stesso genere di letteratura prodotta in Francia. Nei primi due capitoli l'autore mette a confronto le teorie elaborate in Occidente sulla letteratura fantastica e quelle elaborate dagli studiosi di letterature africane (Kane, Abdourahman, Garnier e Doho), cercando di evidenziare ciò che passa, e in che forma, dalle teorie occidentali a quelle concepite per lo studio delle letterature africane. Il confronto impone di superare molte difficoltà, a cominciare dal concetto stesso di fantastico, già problematico in Occidente, ma molto più problematico se applicato al mondo africano, dove il soprannaturale fa parte del quotidiano ed è pertanto inscindibile dal reale, al punto che gli studiosi hanno individuato una "categoria" del fantastico specifica del romanzo realista. Una categoria che ha qualche consonanza con il "realismo magico" di cui ha parlato Daniel Pageaux a proposito della narrativa di Alejo Carpentier (una formula che ha avuto molta fortuna negli studi sulla letteratura caraibica e latinoamericana). Dopo il confronto fra teorie occidentali e teorie "africane", lo studioso passa a individuare i limiti di queste ultime, insistendo sui due più evidenti. Il primo consiste nell'aver dato troppo rilievo allo «choc des civilisations» e dunque al ruolo di rottura della colonizzazione, ruolo che bisognerebbe invece cominciare a ridimensionare per recuperare, sotto la superficie, la continuità con il passato. Il secondo limite è rilevabile nel fatto che «la plupart de ces études portent sur les textes un jugement essentiellement déductif qui empêche de se concentrer sur les données textuelles indispensables à la confrontation dans une perspective comparatiste» (p. 61). I teorici del fantastico africano, secondo Abassolo, hanno dato troppo spazio al fattore antropologico e questo ha impedito una lettura obiettiva del testo, base imprescindibile di una corretta comparazione fra opere provenienti da mondi diversi.

2 L'approccio critico di Abassolo (non mi sembra si possa parlare di una nuova poetica del fantastico africano) è ben sintetizzato da quanto egli stesso afferma nel paragrafo conclusivo del secondo capitolo, intitolato La mise en parallèle des textes français et africains: «Or, quand on sait que les auteurs africains ont lu et bien lu leurs devanciers, il est absurde qu'une influence ne soit perceptible, encore moins une comparaison entre les œuvres des "fantastiqueurs" classiques et celles des auteurs africains. La mise en parallèle directe des textes français et africains que nous envisageons a le mérite de présenter un tableau comparatif plus illustratif puisqu'elle favorise une meilleure visualisation des éléments de comparaison» (p. 65). E gli elementi di comparazione sono, mutatis mutandis, quelli canonici degli studi sulla letteratura fantastica occidentale: spazio, tempo, personaggi, temi, oggetti, costruzione del testo, linguaggio... Il confronto fra gli esiti di questa analisi, fatta a partire dalle stesse componenti ma applicate a prodotti usciti da contesti diversi, aiuta spesso a leggere in un'ottica rinnovata sia il testo di partenza (quello europeo e nello specifico francese) che il testo d'arrivo, quello africano. Ma il procedimento non manca di provocare un certo stupore, e qualche riserva, quando si scopre che gli autori di riferimento europei sono quasi, se non, esclusivamente Mérimée (Mateo Falcone, Colomba, La Vénus d'Ille, Charles XI, La Chambre bleue, etc.), Maupassant (Sur l'eau, Le Horla, La Peur...) e Balzac (La Peau de chagrin), le cui opere sono separate da cento o più anni dagli "omologhi" africani. È innegabile che siano classici della letteratura fantastica francese, ma viene spontaneo chiedersi se tutta l'operazione, fondata su un numero di autori così limitato e su opere tanto lontane nel tempo, non sia costruita su fondamenta un po' fragili. Il tempo di riferimento, sia per quanto concerne le opere che la critica, sembra essere una variabile indipendente nel processo di teorizzazione e di comparazione. 
3 In ogni caso, il lavoro di Abassolo apporta elementi nuovi allo studio del fantastico africano e non mancherà di suscitare, magari proprio in grazia di quelli che sono apparsi ai nostri occhi come dei limiti, puntualizzazioni e nuove indagini, anche comparative, che potranno contribuire alla crescita degli studi in un ambito di ricerca ancora in larga misura da esplorare. 